\title{
Evidence for an Intrinsic Intergap Surface State on GaSb(110) by High-Resolution Angle-Resolved Photoemission
}

\author{
R. Manzke, H. P. Barnscheidt, C. Janowitz, and M. Skibowski \\ Institut für Experimentalphysik, Universität Kiel, D-2300 Kiel, Federal Republic of Germany
}

(Received 6 October 1986)

\begin{abstract}
An intrinsic intergap surface state associated with the occupied dangling-bond state is found on highquality cleaved, $p$-type GaSb(110) surfaces. This conclusion is derived from a high-precision analysis of angle-resolved photoemission data taken with high energy and momentum resolution as well as high absolute accuracy with synchrotron and He I radiation. Thus GaSb is besides GaP the second exception to the accepted rule that III-V compound semiconductors do not have such states.
\end{abstract}

PACS numbers: $73.20 .-\mathrm{r}, 79.60 . \mathrm{Eq}$

It has been well established that surfaces of III-V compound semiconductors are free of surface states in the fundamental energy gap, with the exception of GaP. ${ }^{1}$ For a long time, this agreement was very hard to achieve ${ }^{2-14}$ because of (i) technical insufficiencies in the beginning, i.e., inability to meet the vacuum requirements or other problems associated with performing experiments on semiconducting material of identical highquality standard, ${ }^{11}$ (ii) the preparation of clean, defectfree surfaces which has generated controversy and has remained a problem for over ten years, ${ }^{15}$ and finally (iii) the technical standard of the experimental setups, e.g., for photoemission, which have been applied to this problem.

Many of these problems, especially the technical ones, have been overcome so that, for instance, the preparation of clean semiconductor surfaces is not so critical today. Also, the preparation of good-quality vacuum cleaved (110) surfaces in the case of the III-V compound semiconductors is well known and can be controlled by several techniques, i.e., reflection high-energy electron diffraction. ${ }^{15}$ Furthermore, the angle-resolved photoemission spectroscopy (ARPES) with the high standard now available in several laboratories is a very sensitive probe for surface quality. Intense emission from surface states with narrow linewidths and strong dispersions will be only observed for best-quality, defect-free, clean semiconductor surfaces.

In combination with theory, the experimentally derived electronic structure of the surface can be applied also to investigate the surface geometry ${ }^{16,17}$ which is the essential input for the developed theoretical methods. ${ }^{1,18}$ It is the limits of accuracy of these geometrical input data, usually derived from dynamical LEED studies or, nowadays, also from surface Rutherford backscattering, ${ }^{16}$ which makes a theoretical decision for the existence of intergap surface states, especially for the III-V compound semiconductors with small energy gaps, problematical. Thus, accurate binding energies of surface states and their dispersion should be determined by the now available improved photoemission setups to derive best inputs for the theories.

In this Letter, we report angle-resolved photoemission spectroscopy data of the occupied dangling-bond surface state of $p$-type $\mathrm{GaSb}(110)$, which is found in the fundamental energy gap, $190 \pm 30 \mathrm{meV}$ above the top of the bulk valence band at the $\Gamma_{8}$ point. The spectra were taken with high energy and momentum resolution and absolute accuracy in combination with synchrotron and $\mathrm{He}$ I radiation. The philosophy of this paper is as follows: (i) precise determination of the valence-band maximum at the $\Gamma_{8}$ point from photon-energy-dependent $\left(k_{\perp}\right)$ normal-emission spectra and resolving the uppermost occupied surface state at photon energies, where it is split off the bulk band states; (ii) identification of the surface state as being due to the anion-derived dangling-bond (db) state by determination of its dispersion parallel to the surface $\left(k_{\|}\right)$at different photon energies and its intensity variation with emission angle; (iii) comparison of the position in energy of the $\mathrm{db}$ state with the valenceband maximum $\Gamma_{8}$.

The experiments were carried out on UHV cleaved $p$ type $\left(1.5 \times 10^{17} \text { carriers } / \mathrm{cm}^{3}, \mathrm{Zn}\right)^{19} \mathrm{GaSb}$ single crystals. The orientation and quality were controlled by standard $x$-ray techniques, LEED, and the surface sensitivity of photoemission. Cleavage was performed with a wedge working on both sides of a sawed groove parallel to the (110) surface supported by an adjustable anvil. This stress-free mounting yields best-quality cleaves by application minimum forces. The photoemission spectra were taken with synchrotron radiation from the DORIS II storage ring at Hamburg Synchrotron Radiation Laboratory (HASYLAB) in Hamburg in the region $10 \mathrm{eV}$ $\leq \hbar \omega \leq 30 \mathrm{eV}$ and $\mathrm{He}$ I radiation. The electrons were detected by use of a $180^{\circ}$ spherical analyzer mounted on a goniometer which is movable around two independent axes (improved Leybold Heraeus three-dimensional angle-resolved electron spectrometer). These degrees of freedom allow a very accurate final sample adjustment performed by our taking spectra in small angle steps around the critical points without moving the sample. The capability of this system has been demonstrated else- 
where. ${ }^{20}$ For the spectra shown here an overall energy resolution of $50 \mathrm{meV} \lesssim \Delta E \lesssim 100 \mathrm{meV}$ and an angle resolution of $\pm 0.5^{\circ}$ were chosen.

For the determination of the valence-band maximum of $\mathrm{GaSb}$ we measured energy distribution curves (EDC's) with various photon energies at normal emission $\left(k_{\|}=0\right)$. In Fig. 1 (a) selection of these EDC's close to the valence-band maximum is shown from which we determined the experimental band structure by band mapping for the $\Gamma K X$ direction; the details will be given elsewhere. $^{21}$ The emission peaks assigned to A, B, and C are identified as the bulk $\Sigma$ bands. They reveal significant $\hbar \omega\left(k_{\perp}\right)$ dispersion. At the zone center we found a $\Gamma_{7}-\Gamma_{8}$ spin-orbit splitting of $0.7 \mathrm{eV}$ in good agreement with an empirical pseudopotential calculation ${ }^{22}$ and a previous work. ${ }^{23}$ We found the maximum of the dispersion of the bulk bands (valence-band maximum $\Gamma_{8}$ ) at 23-eV photon energy. ${ }^{21}$

In addition to the bulk-derived peaks we observe a structure for all photon energies on the high-kineticenergy side of the EDC's without any dispersion. This structure which is found to be sensitive to surface contaminations is clearly separated from the bulk bands for photon energies where the bulk bands have already been shifted to lower kinetic energies, for example, at $\hbar \omega=17$ $\mathrm{eV}$, and is approached by the bulk bands near $\Gamma$ (see also Fig. 3). From these experimental facts this peak has to be assigned to a surface state (SS). Its energy position together with that of the bulk-derived state $A$ is plotted as a function of $k_{\perp}$ in the inset of Fig. 1. Note, that the kinetic energy of the state SS is always found to be higher than that of the top bulk state.

In order to investigate whether peak SS is due to the occupied anion-derived dangling-bond state we measured its dispersion parallel to the surface $\left(k_{\|}\right)$in $\bar{\Gamma} \bar{X}^{\prime}$ direction. This is shown for photon energies 17.0 and $21.2 \mathrm{eV}$ in Fig. 2. The dispersion of the surface peak is found to be the same for both photon energies yielding $1.1 \mathrm{eV}$ bandwidth between $\bar{\Gamma}$ and $\bar{X}^{\prime}$, the surface Brillouin-zone boundary. The peak positions of the surface state are plotted for both photon energies as a function of $k_{\|}$in the right inset of Fig. 2, demonstrating that the peaks have the same origin and are localized at the surface without any $k_{\perp}$ dependence.

From the spectra (left inset of Fig. 2) taken with best resolution it can be seen that, when the surface peak has dispersed to lower kinetic energy, there is no emission left in the regime where it is observed in normal emission. This means that there is no indication for the existence of other localized states, e.g., cleavage-induced surface states (extrinsic) or some artifacts resulting from the dopant or contamination effects. These would show no dispersion.

The surface peak SS is clearly attributable to the occupied dangling-bond state. This is further corroborated from the spectra of Fig. 2 taken with momentum $\mathbf{p}$ of the

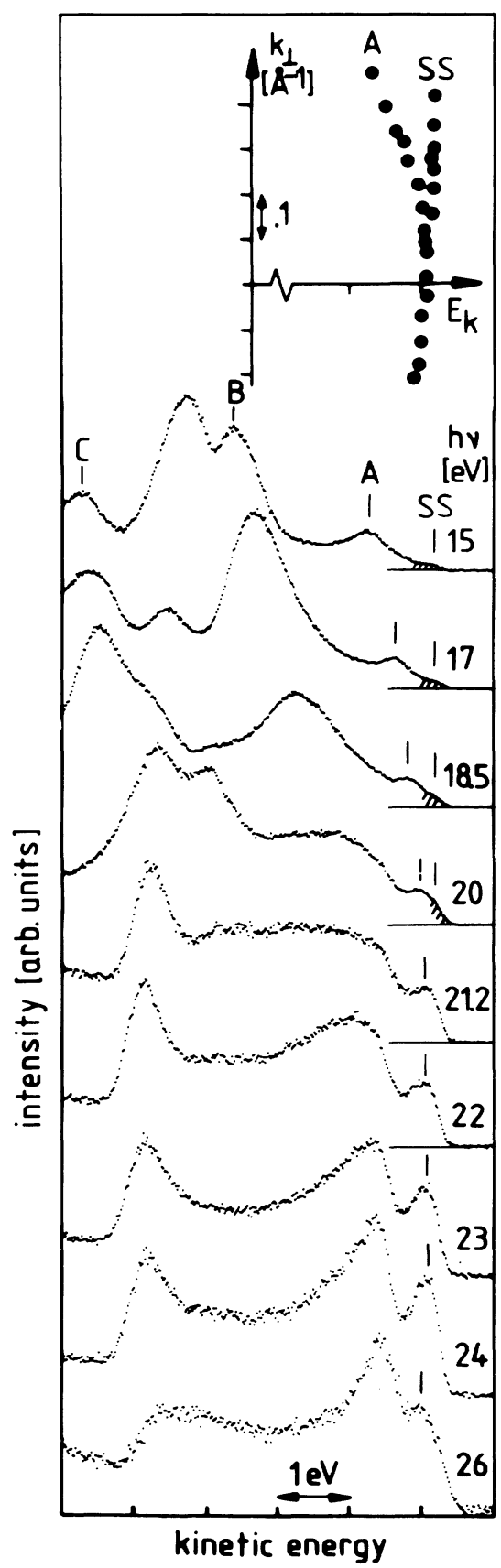

FIG. 1. Normal-emission $\left(k_{\|}=0\right)$ spectra as a function of photon energy. A is the uppermost bulk band, SS is the surface state. Inset: Experimental band structure close to the valence-band maximum of peaks $\mathrm{A}$ and $\mathrm{SS}$ in $\Gamma K X$ direction of the bulk Brillouin zone.

photoelectrons in the direction of the dangling-bond orbital or opposite to it in the $\bar{\Gamma} \bar{X}^{\prime}$ mirror plane. With the assumption of plane-wave final states in first approximation and an atomiclike occupied dangling-bond orbital the emission intensity can be described by the matrix ele- 


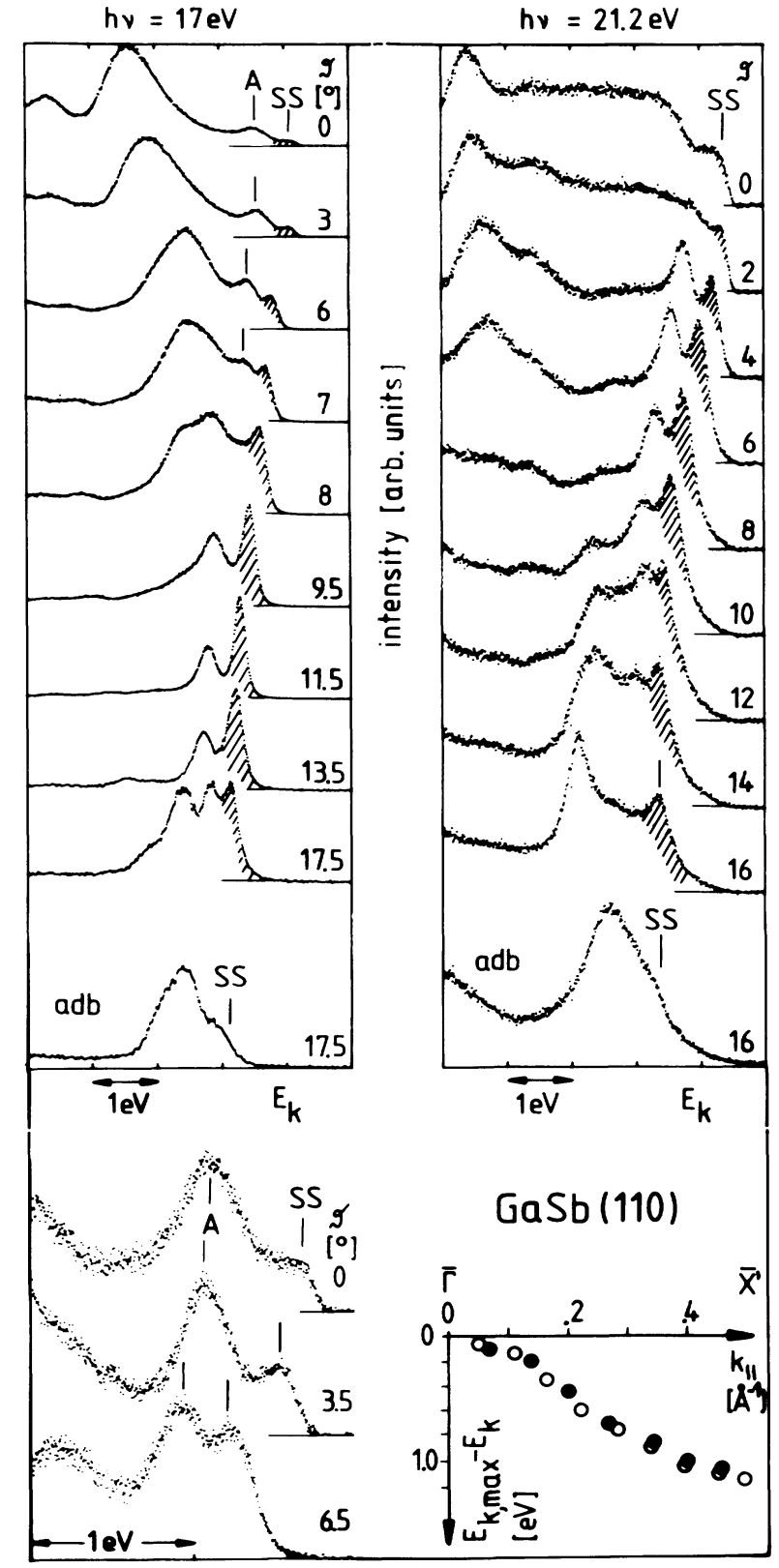

FIG. 2. $k_{\|}$is the dispersion of the surface state at 17- and 21.2-eV photon energy in $\bar{\Gamma} \bar{X}^{\prime}$ mirror plane. Except the spectra indicated by adb (anti-dangling-bond orbital direction) the EDC's are taken in the dangling-bond direction. Right inset: $k_{\|}$vs energy plot of the surface state at photon energies of 17.0 $\mathrm{eV}$ (solid circles) and $21.2 \mathrm{eV}$ (open circles), $E_{k, \max }=\operatorname{maxi}-$ mum kinetic energy at $\bar{\Gamma}$. Left inset: Selection of highestresolution spectra $(\Delta E=50 \mathrm{meV})$ for $17.0-\mathrm{eV}$ photon energy.

ment $^{24}$

$$
\left|\mathbf{A} \cdot \mathbf{M}_{f i}\right|^{2} \sim(\mathbf{A} \cdot \mathbf{p})^{2}|\tilde{\psi}(p)|^{2} .
$$

In the geometry used here the vector potential of the in-

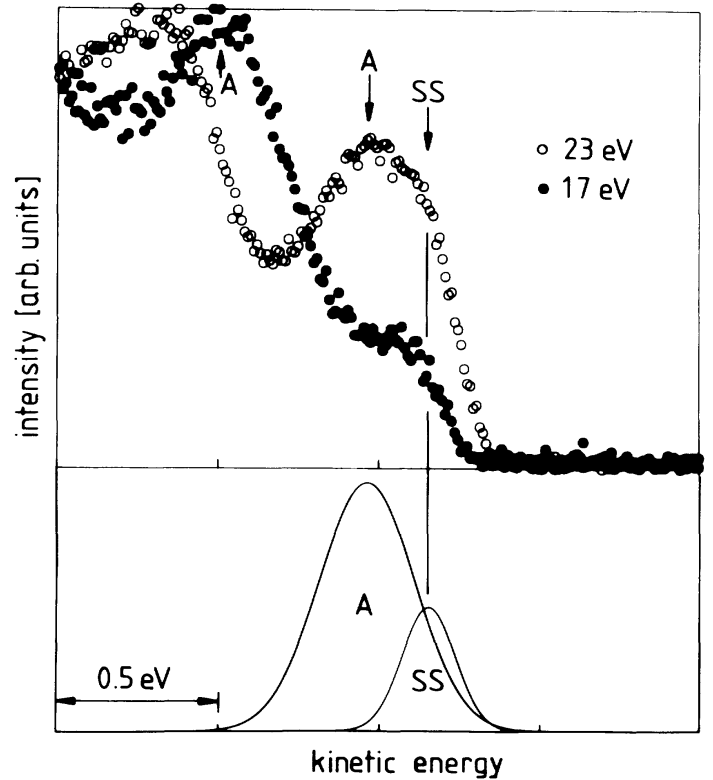

FIG. 3. Comparison of EDC's with photon energy of 23 (where bulk band A has maximum kinetic energy) and $17 \mathrm{eV}$. In the lower part, the Gaussian peaks resulting from a fit of the 23-eV spectrum are shown.

cident light, $\mathbf{A}$, and the sample position, or $\tilde{\psi}(\mathbf{p})$, are kept constant, whereas $\mathbf{p}$, or the position of the analyzer, defines the db or anti-db direction. From the spectra of Fig. 2 the angle dependence is obvious, showing the strong directional character of the db surface state.

By our comparing quantitatively the energy position of the $\mathrm{db}$ state with that of the uppermost bulk band it should be finally decided whether the $\mathrm{db}$ is located in the fundamental gap or not. In Fig. 3 we show the result: high-resolution normal-emission spectra taken at 23- and $17-\mathrm{eV}$ photon energy. As mentioned before, at $17 \mathrm{eV}$ the $\mathrm{db}$ state is found to be clearly separated from the bulk bands, but note that we also observe a weak shoulder on the high-energy side of the bulk band $A$ at $\Gamma_{8}$. The asymmetric peak structure at $\Gamma$ is fitted by two Gaussians shown in the lower part of Fig. 3. An energy difference between the uppermost bulk state $A$ and the dangling-bond state SS of $190 \pm 30 \mathrm{meV}$ is derived.

In conclusion, we found an intrinsic occupied intergap dangling-bond state on the clean (110) surface of GaSb in the center of the Brillouin zone. This has become possible by high energy and angle resolution significantly better than commonly applied. Our findings demonstrate that $\mathrm{GaSb}$ is the second exception besides $\mathrm{GaP}$ to the accepted rule that III-V compound semiconductors do not have such states. Since these surface states are extremely important in the determination of the surface and interface properties of semiconductors, our results call for detailed investigations for other III-V semiconductor compounds as well and should stimulate new refined theoretical calculations concerning the precise en- 
ergy location of the dangling-bond state and its relation to the surface geometry.

The authors would like to thank Professor J. Pollmann, Professor W. Schattke, and I. Schäfer for fruitful discussions. The work was supported by the Bundesministerium für Forschung und Technologie (project No. 50301 ).

${ }^{1}$ See W. Mönch, in Festkörperprobleme, edited by P. Grosse, Advances in Solid State Physics Vol. XXIV (Vieweg, Aachen, 1984), p. 229 and references therein.

${ }^{2}$ J. van Laar and J. J. Scheer, Surf. Sci. 8, 343 (1967).

${ }^{3}$ J. H. Dinan, L. K. Galbraith, and T. E. Fischer, Surf. Sci. 26, 587 (1971).

${ }^{4}$ L. K. Galbraith and T. E. Fischer, Surf. Sci. 30, 185 (1972).

${ }^{5}$ D. E. Eastman and W. D. Grobman, Phys. Rev. Lett. 28, 1378 (1972).

${ }^{6}$ P. E. Gregory, W. E. Spicer, S. Ciraci, and W. A. Harrison, Appl. Phys. Lett. 25, 511 (1974).

${ }^{7}$ D. E. Eastman and J. J. Freeouf, Phys. Rev. Lett. 33, 1601 (1974)

${ }^{8}$ A. Huijser and J. van Laar, Surf. Sci. 52, 202 (1975).

${ }^{9}$ P. W. Chye, I. A. Bababola, T. Sukegawa, and W. E. Spicer, Phys. Rev. Lett. 35, 1602 (1975).

${ }^{10}$ W. Gudat, D. E. Eastman, and J. J. Freeouf, J. Vac. Sci. Technol. 13, 250 (1976).

${ }^{11}$ W. E. Spicer, I. Lindau, P. E. Gregory, C. M. Garner, P. Pianetta, and P. W. Chye, J. Vac. Sci. Technol. 13, 780
(1976).

12 J. van Laar, A. Huijser, and T. L. van Rooy, J. Vac. Sci. Technol. 14, 894 (1977).

${ }^{13}$ A. Huijser, J. van Laar, and T. L. van Rooy, Surf. Sci. 62 , 472 (1977).

${ }^{14}$ H. J. Clemens and W. Mönch, J. Vac. Sci. Technol. 16, 1238 (1979).

${ }^{15}$ F. Cerrina, J. R. Myron, and G. J. Lapeyre, Phys. Rev. B 29, 1798 (1984).

${ }^{16}$ C. Mailhiot, C. B. Duke, and Y. C. Chang, Phys. Rev. B 30, 1109 (1984); D. V. Froelich, M. E. Lapeyre, J. D. Dow, and R. E. Allen, Superlatt. Microstruct. 1, 87 (1985).

${ }^{17}$ U. Aust, R. Manzke, and M. Skibowski, to be published.

${ }^{18}$ See J. Pollmann, in Festkörperprobleme, edited by J. Treusch, Advances in Solid State Physics Vol. XX (Vieweg, Braunschweig, 1980), p. 117; M. Schmeits, A. Maxur, and J. Pollmann, Phys. Rev. B 27, 5012 (1983), and references therein.

${ }^{19}$ MCP Electronic Materials Ltd.

${ }^{20}$ O. Anderson, R. Manzke, and M. Skibowski, Phys. Rev. Lett. 55, 2188 (1985); O. Anderson, G. Karschnick, R. Manzke, and M. Skibowksi, Solid State Commun. 53, 339 (1985).

${ }^{21}$ H. P. Barnscheidt, R. Manzke, and M. Skibowski, to be published.

22 J. R. Chelikowsky and M. L. Cohen, Phys. Rev. B 14, 556 (1976).

${ }^{23}$ T. C. Chiang and D. E. Eastman, Phys. Rev. B 22, 2940 (1980).

${ }^{24}$ N. V. Smith, in Photoemission in Solids $I$, edited by M. Cardona and L. Ley (Springer, Berlin, 1978). 